\title{
Prevalence of Mycobacteremia in Patients With AIDS and Persistant Fever
}

Hélio Arthur Bacha ${ }^{1}$, Sérgio Cimerman ${ }^{1}$, Simone A. de Souza ${ }^{1}$, David Jamil Hadad ${ }^{1}$ and Caio Márcio Figueiredo Mendes ${ }^{2}$

\author{
Instituto de Infectologia Emílio Ribas Infectious Diseases \\ Institute $^{1}$, Department of Infectious Diseases of School of \\ Medicine of São Paulo University ${ }^{2}$, São Paulo, SP, Brazil
}

\begin{abstract}
In the advanced stages of AIDS, characterized by severe immunodepression, tuberculosis (TB) may present with a clinical picture of septic shock, due to typical bacteremia. Hematogenic dissemination of mycobacteria is frequent in immunodepressed patients with $\mathrm{TB}$ or disseminated mycobacteriosis, leading to increased positivity in detection by automated blood culture. The objective of our study was to determine the prevalence of mycobacteremia in patients with AIDS and with prolonged fever seen at the Emilio Ribas Institute of Infectology. Patients with a history of daily fever $\left(\geq 37.8^{\circ} \mathrm{C}\right)$, lasting more than 30 days, and with $\mathrm{CD}_{4}^{+}$helper lymphocyte counts below 200 cells/mL, were selected from February 2001 to March 2002 . A $5 \mathrm{~mL}$ peripheral blood sample was collected from each patient for mycobacterial blood culture by an automated method, using the BACTEC $9000 \mathrm{MB}$ and MB/BACT techniques. Forty-five patients aged on average 35 years, most of them males, were included in the study. The mean $\mathrm{T} \mathrm{CD}_{4}^{+}$lymphocyte count was 58 cells $/ \mathrm{mL}$. Among the samples submitted to blood culture, 30\% gave $M$. tuberculosis growth, with $62 \%$ sensitivity. Among the patients with a negative blood culture, nine had received a diagnosis of TB by another method. Automated blood culture proved to be a technique of relevant diagnostic value for M. tuberculosis in patients with prolonged fever in advanced stages of AIDS. The method is simple, and it helps the physician to select the best therapeutic option.

Key Words: Tuberculosis, automated blood culture, AIDS.
\end{abstract}

A third of the Brazilian population is estimated to be infected with Mycobacterium tuberculosis, with 120,000 new cases occurring every year, 90,000 of which are diagnosed [1]. The states of Rio de Janeiro, São Paulo, Minas Gerais and Bahia have $60 \%$ of all cases, and the impact of this disease is strongly felt in the city of São Paulo, where the mortality due to tuberculosis (TB) increased by $55 \%$ between 1985 and 1995 [2].

Fever has been the most frequent clinical symptom in patients co-infected with HIV and M. tuberculosis (91\%) or with HIV and atypical mycobacteria $(88 \%)[3,4]$.

Received on 12 January 2004; revised 13 July 2004.

Address for correspondence: Dr. Hélio Arthur Bacha. Avenida Paulista, 2073 cj. 1315. Zip code: 01311-940. São Paulo SP, Brazil. Fax: (55 11) 251-2084. Phone: (55 11) 251-4120.

E-mail: hbacha@terra.com.br

The Brazilian Journal of Infectious Diseases 2004;8(4):290-295 (C) 2004 by The Brazilian Journal of Infectious Diseases and Contexto Publishing. All rights reserved.
The clinical manifestations of $\mathrm{TB}^{\text {in }} \mathrm{HIV}^{+}$patients depend on the degree of immunodeficiency of each patient [5]. Those with $\mathrm{CD}_{4}^{+}$counts above 300 cells/mm3 behave clinically like immunocompetent patients, whereas patients with $\mathrm{CD}_{4}^{+}$counts below 200 tend to present a nonspecific clinical pattern [6], which may manifest as bacteremia and septic shock $[7,8]$.

The diagnosis of TB is even more difficult and time consuming [9] in immunodepressed patients, because of the similarity to the clinical presentation of various opportunistic diseases, with the risk of progression of the base disease; it is not uncommon to make the initial diagnosis of disseminated TB post mortem [10-12].

In confirmed cases of pulmonary $\mathrm{TB}$ in $\mathrm{HIV}^{+}$ patients with $\mathrm{CD}_{4}^{+}$lymphocyte counts of less than 200 cells/mL, blood cultures are positive for Koch's bacillus in about $40 \%$ of cases [13].

In underdeveloped countries, M. tuberculosis has been observed to be the most common agent in clinical 
manifestations of bacteremia occurring in non-hospital community environments $[14,15]$.

We investigated the prevalence of mycobacteremia in patients with AIDS and with persistent fever (a history of a temperature $\geq 37.8^{\circ} \mathrm{C}$ for more than 30 days) at a reference hospital for infectious diseases in the city of São Paulo.

\section{Materials and Methods}

\section{Patients}

From March 2001 to February 2002, we selected all patients attended at the First Aid (FA) station and on the wards of the Emilio Ribas Institute of Infectology (Instituto de Infectologia Emílio Ribas, IIER) who were older than 18 years and who had a positive serologic diagnosis of HIV I/II infection determined by an immunoenzymatic test (ELISA) and confirmed by a specific Western blot, plus a history of daily fever $\left(\geq 37.8^{\circ} \mathrm{C}\right)$ for more than 30 days and $\mathrm{CD}_{4}^{+}$helper lymphocyte counts below 200 cells/mL blood. The patients were selected by an active daily search at the FA station, and by periodic visits to the wards of IIER. We did not select the patients by the criterion of fever of undetermined origin (FUO) of Petersdorf [16,17], which is based on the concept of fever of duration of more than 21 days after clinical, radiological and laboratory investigation for 7 days [18]. Our concept of persistent fever for more than 30 days concerns the clinical situation of the patient at the time of presentation at the IIER, i.e., a history of fever of 30 days duration or more, regardless of whether there was a radiological or laboratory investigation [19]. Exclusion criteria were: age of less than 18 years, a diagnosis of TB made less than one year before, a previous diagnosis of TB, a previous diagnosis of mycobacteriosis, non-adherence to treatment with TB-static schemes, and previously investigated cases with positive blood cultures for any type of mycobacteria.

The study was approved by the Ethics Research Committees of the University Hospital, Faculty of Medicine, University of São Paulo (USP) and of IIER.
All patients selected signed a term of informed consent to participate in the study.

\section{Methods}

A 5-mL peripheral blood sample was collected from each patient for mycobacterial culture using an automated method. The samples from 16 patients were analyzed in an MB/blood culture flask specific for mycobacteria and the result was read with an automated MB/Bact system (Organon-Teknika). The samples of the other 29 patients were collected by the same technique but inoculated into a Bactec/Myco F flask, also specific for mycobacteria, and analyzed with an automated continuous monitoring system (BACTEC) [20]. The performance and operational characteristics of the two systems are similar. The samples found to be positive by automated analysis were submitted to identification of the mycobacterial complex using a DNA probe (Accuprobe, Amplified Mycobacterium Tuberculosis Direct Test Accuprobe ${ }^{\circledR}$ [Gen-Probe, Inc]). This test is specific for the identification of mycobacterium species within a time not exceeding two hours, with excellent sensitivity $(100 \%)$ and with $99.2 \%$ specificity $[21,22]$.

\section{Results}

Forty-five patients fulfilled the criteria for inclusion in the study and the result of blood culture could not be obtained in only one case. The mean age of the sample was 35 years, ranging from 21 to 59 years, with a median of 33 years. Twenty-nine patients $(64 \%)$ were males and $16(36 \%)$ were females.

Regarding anti-retroviral medication, only 5 of the patients (11\%) were adhering to regular treatment, 27 $(60 \%)$ had never taken this type of medication, 11 $(24 \%)$ reported an irregular use or abandonment of treatment due to problems with side effects, and for two patients $(4 \%)$ it was not possible to obtain information about the use of specific medications.

The patients were severely immunodepressed, with $34(76 \%)$ showing $\mathrm{CD}_{4}^{+}$lymphocyte counts of less 
than 100 cells/mL and only $11(24 \%)$ showing $\mathrm{CD}_{4}^{+}$ lymphocyte counts between 100 and 200 cells $/ \mathrm{mL}$. Mean $\mathrm{CD}_{4}^{+}$count was 58 cells $/ \mathrm{mL}$.

One patient had no blood sample processed but had a diagnosis of TB by biopsy. Sixteen of the selected patients had a blood sample analyzed in an MB/blood culture flask specific for mycobacteria, with a reading taken with an MB/Bact automated system (OrganonTeknika). The other 28 patients provided a blood sample collected by the same technique but inoculated into a Bactec/Myco Ffals, also specific for mycobacteria, and analyzed with a continuous monitoring automated system (BACTEC).

Mycobacterial growth was observed in 13 of the 44 samples processed (30\%) (Table 1). Eight of the patients with a negative blood culture received a diagnosis of TB by a method other than blood culture. In our patient sample, the sensitivity of blood culture was $62 \%$ (Figure 1).

\section{Discussion}

Although the introduction of new antiretroviral drugs in the mid-nineties was an important change in terms of improved quality of life and increased longevity of HIVinfected patients, many such patients continue to progress to severe immunodepression [23].

Only a small fraction of the patients studied here used anti-retroviral medication in a correct manner (11\%); 60\% had never taken any specific medication. This fact is explained by the difficulty that a significant number of patients have in adhering to a therapeutic scheme that requires the continuous daily ingestion of multiple medications at fixed times, with strong side effects [24]. There are also patients who are not aware of their conditions of risk for infection and who were already in a situation of advanced immunodepression when they received a serological diagnosis of HIV. This situation caused a large proportion of the patients, three quarters of them, to have $\mathrm{CD}_{4}^{+}$lymphocyte counts below 100 cells $/ \mathrm{mL}$. The frequent occurrence of mycobacteremia in this group of patients was due to their state of severe immunodepression.
The higher proportion of males (64\%) must reflect the gender distribution among $\mathrm{HIV}^{+}$patients attended at the IIER, since the risk to develop TB among $\mathrm{HIV}^{+}$ patients is the same for both sexes [25].

The preponderance of TB as a cause of prolonged fever in patients with AIDS was clear in the present sample. Of the 44 patients studied, 22 (49\%) had a diagnosis of TB and in $13(30 \%)$ cases the diagnosis of $M$. tuberculosis bacteremia was confirmed by growth in specific automated blood culture, demonstrating the prevalence of this type of infection in our study.

The use of two different products for automated blood culture did not interfere with our results since, according to the experience of bacteriologists, Bactec/ Myco F and MB/Bact have equivalent sensitivity. The sensitivity of automated blood culture observed in our sample (62\%, Table 1) demonstrates that this is a useful complementary method for the identification of disseminated TB in immunocompetent patients, and that it should be applied to immunodepressed patients with a suspicion of disseminated TB before starting empirical treatment. In our calculation of sensitivity, all patients with a diagnosis of TB formed the numerator, as if all of them had potentially detectable bacteremia. Thus, it is possible that this number was even higher.

In our study, if we had not limited blood collection to a single sample per patient for blood culture examination we would not have appreciably improved our sensitivity. Fandinho [26], in a study in which three blood samples were collected for mycobacterial culture from 50 patients with AIDS with a clinical suspicion of TB or disseminated mycobacteriosis, obtained concordant results in $39(78 \%)$ cases. In that study, published in a bulletin of the World Health Organization, the author stated that his data supported the recommendation of testing only one blood sample per patient, and he recommended automated blood culture for mycobacteria [26].

Among the patients in our series, there was no case of identification of a mycobacteriosis caused by any atypical mycobacteria identified by blood culture.

Bamman et al. [27] conducted a survey of the exams of all samples for mycobacterial culture in the 
Table 1. Sensitivity profile of automated blood culture for mycobacteria in febrile patients with AIDS at the Emilio Ribas Infectology Institute, 2002

\begin{tabular}{lcc}
\hline Blood culture for mycobacteria & N & \% \\
\hline Negative with a diagnosis of TB & 8 & 38 \\
Positive & 13 & 62 \\
Total number of TB cases submitted to blood culture & 21 & 100,00 \\
\hline
\end{tabular}

Figure 1. Prevalence of mycobacteremia in patients in an advanced stage of AIDS at a reference hospital in the city of São Paulo, Brazil.

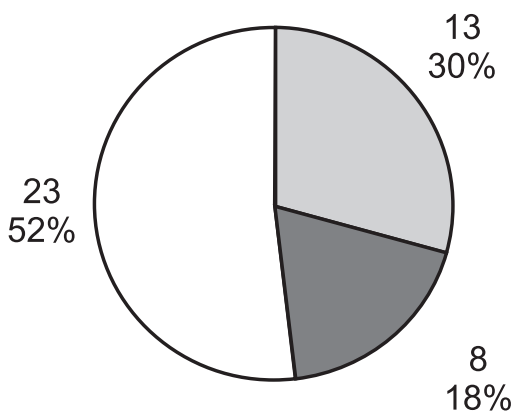

Blood culture positive for M. Tuberculosis

TB with negative blood culture

Non-TB negative bood culture

same bacteriology section for a total of 6,781 exams of respiratory samples (sputum, bronchoalveolar lavage, gastric juice, and pulmonary biopsies obtained by open surgery) performed in 2000 and 2001 and reported positivity and identification of mycobacterial species with 574 mycobacterial strains being identified. Of these, 112 (19.5\%) were "non-tuberculosis mycobacteria", with four cases being also concomitantly positive for $M$. tuberculosis. We calculated the occurrence of 466 (81.2\%) M. tuberculosis strains. Perhaps the fact that these were respiratory samples and that patients with respiratory symptoms had already been submitted to a first diagnostic screening by bacilloscopy, not necessarily with species identification by culture, would explain the higher proportion of atypical mycobacteria observed in the study by Bamman et al. [27].

We also did not confirm the finding of a large number of atypical mycobacteria reported by Barreto et al.[28] who, in a study of patients with persistent fever and pancytopenia, detected M. avium/intracellulare in $18.4 \%$ of patients and M. tuberculosis in only $7.2 \%$. Published in 1993, this study has been widely cited in the medical literature, since until then the belief was 
that atypical mycobacteria would not be numerically important in underdeveloped countries, where there is a high prevalence of $M$. tuberculosis infection as a cause of the consumptive diseases occurring in AIDS patients. The local influence of that study on the clinical therapeutic conduct for patients with AIDS and persistent fever was also significant since the drug scheme for the treatment of mycobacteria is specific for each species. We believe that in the study by Barreto the importance of mycobacteria was overestimated due to a sampling bias, since the patients were selected among those referred for specialized hematology evaluation.

Our study also has limitations. The sample that we selected only includes patients who use the services of IIER. As is the case for all prevalence studies, the representativeness of the sample is limited to the sample itself.

Our results may reflect the fact that the sample consisted of patients with a low socioeconomic level since it was selected at a hospital that provides support for the public network, whose services are not habitually used by the population of higher income. This observation is important, because one of the determinants of infection with $M$. tuberculosis is the low socioeconomic condition of the patients [29,30].

The fact that we did not detect the growth of atypical mycobacteria in blood culture does not permit us to infer that none of these species are important as a cause of prolonged fever in febrile patients. The method of transverse epidemiological investigation does not have a statistical sensitivity that would permit us to reach this conclusion.

The greater importance of M. tuberculosis in relation to other mycobacteria among patients with AIDS in Brazil agrees with the numbers obtained in a survey conducted at the bacteriology section of the IIER laboratory by Souza S.A. and Barletti S.C. (personal communication), which showed that 1455 blood cultures for mycobacteria were processed in 2001, with 116 of them ( $8 \%$ ) being positive, with the following distribution: 101 cases of $M$. tuberculosis $(87 \%)$ and 15 cases of $M$. avium/intracellulare (13\%).
The number of positive blood cultures for $M$. tuberculosis (13 cases), added to the number of patients with a diagnosis of TB despite a negative blood culture ( 9 cases), results in a total of $22(49 \%)$ patients with a diagnosis of TB among the 45 patients selected. This number leads us to infer that, in our sample, TB is the main cause of persistent fever in patients with AIDS.

We conclude that blood culture for mycobacteria using an automated method is an important diagnostic tools for patients with AIDS and with persistent fever, being of help in the planning of the specific conduct for each individual. We also suggest that additional more expanded studies of a multicenter nature should be carried out to demonstrate in a more definitive manner the importance of each mycobacterium in patients with AIDS and with persistent fever.

\section{References}

1. Ruffino Netto A. Informe em palestra no I Encontro Panamericano de Tuberculose - Campos de Jordão SP, 1998.

2. Athias G. Mortes por tuberculose aumentam $55 \%$. Folha de S. Paulo; 11 dez 2000:1.

3. Modilevsky T., Sattler F.R., Barnes P.F. Mycobacterial disease in patients with human immunodeficiency virus infection. Arch Inter Med 1989;149:2201-5.

4. Kramer F., Modilevsky T., Waliany A.R., et al. Delayed diagnosis of tuberculosis in patients with human immunodeficiency virus infection. Am J Med 1990; $89: 451-6$.

5. Jones B.E., Houng S.M.M., Antoniskis D., et al. Relationship of the manifestations of tuberculosis to CD4 cell count in patients with human immunodeficiency virus infection. Am Rev Respir Dis 1993; 148:1292-7.

6. Porter J.D.H. Mycobacteriosis and HIV infection: the new public health challenge. J Antimicrob Chemother 1996;37(S):113-20.

7. Archibald L.K., den Dulk M.O., Pallangyo K.J., Reller L.B. Fatal Mycobacterium tuberculosis bloodstream infections in febrile hospitalized adults in Dar es Salaam, Tanzania. Clin Infect Dis 1998;290-6.

8. Clark T.M., Burman W.J., Cohn D.L., Mehler O.S. Septic shock from Mycobacterium tuberculosis after therapy for Pneumocystis carinii. Arch Intern Med 1998; $158: 1033-5$. 
9. Cobo J., Oliva J., Asensio A., et al. Predicting tuberculosis among HIV-infected patients admitted to hospital: comparison of a model with clinical judgment of infectious disease specialists. Eur J Clin Microbiol Infect Dis 2001;20:779-84.

10. Borges A.S., Ferreira M.S., Nishioka A.S., et al. Agreement between premortem and postmortem diagnosis in patients with acquired immunodeficiency syndrome observed at a Brazilian teaching hospital. Rev Inst Med Trop São Paulo 1997;39:417-21.

11. Flora G.S., Modilevsky T., Antoniskis D., Barnes P.F. Undiagnosed tuberculosis in patients with human immunodeficiency virus infection. Chest 1990;98:1056-9.

12. Gutierrez E.B. Influência da infecção pelo vírus de imunodeficiência humana. São Paulo;2002. Dissertação de mestrado Faculdade de Medicina da USP.

13. Cernoch P.L., Enns R.K., Saubolle M.A., Wallace Jr R.C. Laboratory diagnosis of the mycobacterioses cumulative techniques and procedures in clinical microbiology 16A. Am Soc Microbiol 1994.

14. McDonald L.C., Archibald L.K., Rheanpumikankit S., et al. Unrecognised Mycobacterium tuberculosis bacteraemia among hospital inpatients in less developed countries. Lancet 1999;354:1159-63.

15. Archibald L.K., den Dulk M.O., Pallangyo K.J., Reller L.B. Fatal Mycobacterium tuberculosis bloodstream infections in febrile hospitalized adults in Dar es Salaam, Tanzania. Clin Infect Dis 1998;290-6.

16. Hirschmann J.V. Fever of unknown origin in adults. Clin Infect Dis 1997;24:291-302.

17. Petersdorf R.G. Fever of unknown origin, an old friend revisited. Arch Intern Med 1992;152:21-2

18. Arnow P.M., Flaherty J.P. Fever of unknown origin. Lancet 1997;350:575-80.

19. Fever of unknown origin in the HIV-infected patient in Gantz NM Manual of Clinical Problems in Infectious Disease, 4th ed. Philadelphia: Lippincott Williams \& Williams 1999:478-81.

20. Metchock B.G., Nolte F.S., Wallace Jr R.J. Mycobacterium in. Murray's Manual of Clinical Microbiology.Washington DC. ASM Press 1999; 399:436.

21. Musial C.E., Tice L.S., Stockman L., Roberts G.D. Identification of mycobacteria from culture by using the Gen-Probe Rapid Diagnostic System for Mycobacterium avium complex and Mycobacterium tuberculosis complex. J Clin Microbiol 1988;26:2120-3.

22. Gen-Probe. Accuprobe instructions. Available at http:// www.gen-probe.com nov 2002.

23. Palella Jr F.J., Delaney K.M., Moorman A.C., et al. Declining morbidity and mortality among patients with advanced human immunodeficiency virus infection. N Engl J Med 1998;338:853-60.
24. Nemes M.I.B., Souza M.F.M., Kalichman A.O., et al. Prevalência da aderência e fatores associados. Available at http://www.AIDS.gov.br/final/biblioteca/avalia1/ relat2.htm, dez 2002.

25. Swaminathan S., Ramachandran R., Baskaran G., et al. Risk of development of tuberculosis in HIV-infected patients. Int J Tuberc Lung Dis 2000;4:839-44.

26. Fandinho F.C., Grinsztejn B., Veloso V.G., et al. Diagnosis of disseminated mycobacterial infection: testing a simple and inexpensive method for use in developing countries. Bull World Heath Organ 1997;75:361-6.

27. Bammann R.H., Vázquez C.M.P., Haddad D.J., et al. Micobactérias não tuberculosas isoladas em amostras respiratórias de patients acompanhados no Instituto de Infectologia Emílio Ribas (IIER). J Pneumol 2002;28:S108.

28. Barreto J.A., Palaci M., Ferrazoli L., et al. Isolation of Mycobacterium avium complex from bone marrow aspirates of AIDS patients in Brazil. J Infect Dis 1993; $168: 777-9$.

29. Barnes P.F., El-Hajj H., Preston-Martin S., et al. Transmission of tuberculosis among the urban homeless. JAMA 1996;275:305-7.

30. Waaler HT. Tuberculosis and poverty. Int J Tuberc Lung Dis 2002;6:745-6. 